Revue d'histoire de l'Amérique française

REVUE D.HISTOIRE DE L'AMÉRIQUE FRANÇAISE

\title{
Officiers et marchands : les sociétés de commerce des fourrures, 1715-1760
}

\section{Gratien Allaire}

Volume 40, numéro 3, hiver 1987

URI : https://id.erudit.org/iderudit/304469ar

DOI : https://doi.org/10.7202/304469ar

Aller au sommaire du numéro

\section{Éditeur(s)}

Institut d'histoire de l'Amérique française

\section{ISSN}

0035-2357 (imprimé)

1492-1383 (numérique)

Découvrir la revue

Citer cet article

Allaire, G. (1987). Officiers et marchands : les sociétés de commerce des fourrures, 1715-1760. Revue d'histoire de l'Amérique française, 40(3), 409-428. https://doi.org/10.7202/304469ar
Résumé de l'article

L'analyse de 202 contrats notariés de société pour le commerce des fourrures donne, de cet aspect du commerce, un portrait plus précis que celui que l'on a tiré de la correspondance administrative. Mise en place et rodée à partir de 1715 , cette forme d'exploitation prend toute son importance entre 1722 et 1734, pour décliner ensuite. Au cours de ces 8 ans, les officiers et les commandants de poste s'associent à des marchands-voyageurs pour l'exploitation des postes. Alors que les premiers apportent à la société le droit de faire la traite, les seconds se chargent de la gestion des affaires. Quant aux marchands-équipeurs, ils approvisionnent ces sociétés sans en faire partie. 


\title{
OFFICIERS ET MARCHANDS: LES SOCIÉTÉS DE COMMERCE DES FOURRURES, 1715-1760 ${ }^{1}$
}

\author{
GRATIEN ALLAIRE \\ Faculté Saint-Jean \\ Université de l'Alberta
}

\begin{abstract}
RÉSUMÉ
L'analyse de 202 contrats notariés de société pour le commerce des fourrures donne, de cet aspect du commerce, un portrait plus précis que celui que l'on a tiré de la correspondance administrative. Mise en place et rodée à partir de 1715 , cette forme d'exploitation prend toute son importance entre 1722 et 1734 , pour décliner ensuite. Au cours de ces 8 ans, les officiers et les commandants de poste s'associent à des marchands-voyageurs pour l'exploitation des postes. Alors que les premiers apportent à la société le droit de faire la traite, les seconds se chargent de la gestion des affaires. Quant aux marchands-équipeurs, ils approvisionnent ces sociétés sans en faire partie.
\end{abstract}

\begin{abstract}
A total of 202 contracts of partnership for the fur trade have been found in the notarial archives. The analysis of their content results in a portrait of this aspect of the trade which is more detailed than the one usually derived from the official correspondance. Although in use since 1715, this form of exploitation played it most important role from 1722 to 1734, and declined subsequently. During the peak years, posts commanders and officers formed partnerships with marchands-voyageurs in order to exploit the posts. The former brought the partnership the right to trade; the latter managed the business activities. A third group, the marchands-équipeurs, acted as suppliers of the company without directly participating in it.
\end{abstract}

Au cours des dernières années, les chercheurs ont accordé une importance accrue au commerce des fourrures et à ses divers aspects, en particulier à ses incidences sociales. Profitant du déménagement des archives de la Compagnie de la baie d'Hudson de Londres à Winnipeg et de leur plus grande accessibilité, les historiens ont fait porter leurs recherches sur l'Ouest du Canada et, entre autres, sur les sociétés

1 Ce texte a fait l'objet d'une communication au Congrès annuel de l'Institut d'histoire de l'Amérique française, le 19 octobre 1985. La recherche sur laquelle il est basé a été faite alors que l'auteur bénéficiait d'un congé de l'Université de l'Alberta et d'une subvention de recherche du Conseil de recherches en sciences humaines du Canada. L'auteur tient à remercier Claude Desrosiers et Claire Joron pour leur participation au dépouillement des contrats notariés, le professeur José Igartua pour l'accès à son fichier sur les années 1750-1760 et ses suggestions relatives au texte, de même que le professeur John Dickinson pour ses commentaires.

[409]

RHAF, vol. 40, no 3, hiver 1987 
amérindiennes $^{2}$, sur les opérations de la Compagnie de la baie d'Hudson $^{3}$, sur le rôle et la place des femmes amérindiennes dans les échanges commerciaux ${ }^{4}$ et sur le phénomène du métissage résultant de la rencontre des deux sociétés ${ }^{5}$.

Le commerce des fourrures basé à Montréal n'a pas été l'objet d'un renouveau d'intérêt aussi prononcé. Des recherches récentes ont jeté un éclairage neuf sur les opérations de la Compagnie du NordOuest ${ }^{6}$. Louise Dechêne a prêté une attention particulière aux divers aspects du commerce à la fín du $17 \mathrm{e}$ siècle et au début du $18 \mathrm{e}$ siècle: organisation, personnel, marchandises et niveau de profits ${ }^{7}$. Les engagés de la traite de la première moitié du $18 \mathrm{e}$ siècle ont fait l'objet d'une analyse détaillée à partir des contrats d'engagement ${ }^{8}$. Pour le reste, l'étude de Harold A. Innis demeure, malgré la critique qu'en a faite récemment W. J. Eccles ${ }^{9}$, l'ouvrage essentiel sur le commerce des fourrures basé à Montréal ${ }^{10}$. De nombreux aspects de cette activité économique et de son organisation complexe restent donc mal connus.

Parmi ces facettes, se trouve un aspect de l'organisation du commerce: les sociétés de commerce des fourrures ou, plus précisément, les sociétés pour l'exploitation des postes des Pays-d'en-haut. Peu d'historiens ont écrit sur ces sociétés et sur le rôle des associés. Sauf exception, leur présentation du sujet résulte de l'étude de sources secondaires et paraît trop statique. H. A. Innis a décrit un type d'association qui eut cours vers la fin du Régime français: «a type of organization in which profit sharing or partnership developed between the trader in the interior and the Montreal merchant. ${ }^{11}$ Selon l'économiste-historien, le contrôle de la société, en situation de concurrence, avait tendance à être entre les mains du «trader»; mais, en situation de monopole, le rôle de ce dernier ressemble plus à celui d'un gérant. Cameron Nish, pour sa

2 A. J. Ray, Indians in the Fur Trade; Their Role as Trappers, Hunters, and Middlemen in the Lands Southwest of Hudson's Bay, 1660-1870 (Toronto, University of Toronto Press, 1974).

Arthur J. Ray and Donald B. Freeman, «Give Us Good Measure»: An Economic Analysis of Relations Between the Indians and the Hudson's Bay Company Before 1763 (Toronto, University of Toronto Press, 1978).

4 Sylvia VanKirk, "Many Tender Ties»: Women in Fur-Trade Society in Western Canada, 1670-1870 (Winnipeg, Watson \& Dwyer Publishing, 1980).

5 Jennifer S. H. Brown, Strangers in Blood: Fur Trade Company Families in Indian Country (Vancouver, University of British Columbia Press, 1980).

6 Quelques-unes des communications présentées lors de la Cinquième Conférence nordaméricaine sur la traite des fourrures portaient sur le sujet.

7 Louise Dechêne, Habitants et marchands de Montréal au XVIIe siècle (Montréal, Plon, 1974).

Gratien Allaire, Les engagés de la fourrure, 1701-1745: une étude de leur motivation. Thèse de Ph.D., Université Concordia, 1982.

9 W. J. Eccles, «A Belated Review of Harold Adams Innis, The Fur Trade in Canada», Canadian Historical Review, 60,4 (Dec. 1979): 419-441.

10 Harold A. Innis, The Fur Trade in Canada (Toronto, University of Toronto Press, 1970).

11 Ibid., 112. 
part, a écrit qu'un «groupe restreint d'officiers et de marchands dominaient la traite des fourrures en Nouvelle-France pendant les années 1729 à $1748 »^{12}$, sans préciser le rôle de chacun. W. J. Eccles place le contrôle du commerce entre les mains des officiers militaires ${ }^{13}$ ou, dans un écrit plus récent, à un groupe restreint de bourgeois ${ }^{14}$. Quant à Louise Dechêne, si elle fait état de trois groupes de partenaires, elle ne s'étend pas sur leurs rôles respectifs.

Ce texte a pour but d'établir avec plus de précision l'organisation de ces sociétés qui se chargeaient de l'exploitation des postes de l'Ouest et de faire voir les variations qui s'y trouvent. L'analyse porte sur les années 1715 à 1760 , période qui commence avec le retour au système des congés et le rétablissement subséquent des postes de l'Ouest et qui se termine avec la fin du Régime français. La documentation utilisée, les contrats de société, permet de remettre en question la présentation habituelle de ces sociétés et du rôle des associés dans le commerce des fourrures. L'étude de ces documents notariés permet d'identifier les deux principaux groupes de participants à ces sociétés, les officiers des postes et les marchands-voyageurs. Les premiers apportent le privilège du commerce; les seconds mettent à profit leur expertise et se chargent de l'exploitation du poste. A ces deux groupes s'en greffe un troisième, les marchands-équipeurs, dont le rôle consiste à fournir les marchandises de traite et à agir comme procureurs de la société ou de ses membres. Il arrive rarement qu'ils fassent partie de la société de commerce.

\section{LA DOCUMENTATION UTILISÉE}

Notre analyse des partenaires du commerce des fourrures se fonde essentiellement sur une documentation tirée des greffes des notaires. Il s'agit, d'abord et avant tout, de contrats de société qui, en liant les parties, déterminaient les fonctions de chacune. A ces contrats s'en ajoutent divers autres, qui ont servi à mieux délimiter le rôle des divers partenaires et à mieux établir l'évolution de l'ensemble. Ce sont des cessions et baux à ferme de poste, des conventions de fourniture de marchandises, des dissolutions de société et un nombre élevé de procurations.

12 Cameron Nish, Les Bourgeois-Gentilshommes de la Nouvelle-France, 1729-1748 (Montréal, Fides, 1968), 98.

13 «The officers who obtained posts formed companies, consisting mainly of relatives, to raise the capital and handle the trade.» W. J. Eccles, The Canadian Frontier (Albuquerque, University of New Mexico Press, 1983), 147, revised edition.

${ }_{14}$ "As the 18th century wore on and costs rose with distance, the trade came to be controlled by a small number of bourgeois (...) Most companies consisted of 3 or 4 men who obtained from the authorities the lease on the trade at a specific post for 3 years; all members shared profits or losses proportional to the capital subscribed. Trade goods were usually obtained on credit, at $30 \%$ interest, from a small number of Montréal merchants who marketed the furs through their agents in France.» W. J. Eccles, «Fur Trade» in James H. Marsh, ed., The Canadian Encyclopedia (Edmonton, Hurtig Publishers, 1985), 705. Cet extrait de l'article de Eccles reflète bien l'état actuel des connaissances relatives au commerce des fourrures basé à Montréal au 18e siècle. 
Jacques Savary des Brûlons, l'autorité en matière de commerce pour le 18e siècle, définit ainsi la société d'affaires: «Contrat, acte ou traité qui se fait entre deux ou plusieurs personnes, par lequel elles se lient ensemble pour un certain tems, \& conviennent de se communiquer les profits, \& supporter également les pertes qui se feront dans les affaires pour lesquelles la Société est contractée.» ${ }^{15}$ Il distingue trois types de sociétés: les sociétés générales (ou collectives ou ordinaires), les sociétés en commandite et les sociétés anonymes. La première réunit «deux ou plusieurs Marchands, qui agissent tous également pour les affaires de la Société, \& qui font le négoce sous leurs noms collectifs, qui sont connus de tout le monde». La seconde se fait entre deux personnes «dont l'une ne fait que mettre son argent dans la Société sans faire aucune fonction d'Associé; \& l'autre, que l'on nomme en termes mercantiles le Complimentaire de la société, donne quelquefois son argent, mais toujours son industrie, $\&$ fait le commerce sous son nom des choses dont ils sont convenus ensemble». La troisième, la société anonyme, «ne se fait sous aucun nom, mais (...) tous les Associés travaillent chacun en leur particulier, sans que le Public soit informé de leur Société» ${ }^{16}$.

L'ordonnance du commerce ${ }^{17}$ de mars 1673 , qui ne fournit aucune indication sur les sociétés anonymes ${ }^{18}$, stipule que sociétés générales et sociétés en commandite doivent être rédigées par écrit, sous seing privé ou devant notaire ${ }^{19}$. Elle ajoute que l'extrait doit en être enregistré au greffe approprié et inséré dans un tableau exposé en un lieu public. Si cette ordonnance a été en vigueur en Nouvelle-France, elle n'a pas laissé de trace: aucun registre de sociétés n'a été retrouvé. Cependant, on peut considérer que son contenu a beaucoup influencé ce qui se faisait dans la colonie à ce sujet, du moins en ce qui concerne le contenu des contrats ${ }^{20}$. Le dépouillement des minutes notariales ne donne pas nécessairement toutes les sociétés de traite. Comme il n'était pas requis que les contrats de société soient reçus par un notaire, plusieurs d'entre

15 Jacques Savary des Brûlons, Dictionnaire universel de commerce... (Paris, 1748, tome 3 , col. 144).

16 Ibid.

17 Cette ordonnance touche plusieurs sujets relatifs au commerce: l'apprentissage, la tenue de livres, les lettres et les billets de change, les contraintes par corps, les séparations de biens, les cessions de biens, les faillites et les banqueroutes, la juridiction des consuls et les sociétés. Isambert, DeCrusy et Taillandier, Recueil général des anciennes lois françaises, depuis l'an 420, jusqu'à la révolution de 1789 (Paris, 1829), 19: 92-107.

${ }_{18}$ Selon Savary des Brûlons, ce type de société est réprouvé par les ordonnances. Le simple fait que l'ordonnance de mars 1673 n'en parle pas peut être considéré en soi comme une réprobation. ch. 1, p. 6 .

Savary des Brûlons, Dictionnaire, 3, col. 144; Le Parfait Négociant, partie II, livre I,

Selon José E. Igartua, le fait que l'ordonnance ne semble pas avoir été enregistrée par le Conseil souverain n'entame pas nécessairement sa validité. The Merchants and Négociants of Montréal, 1750-1775: A Study in Socio-Economic History. Thèse de Ph.D., Michigan State University, 1974, 130n. 
eux furent passés sous seing privé; quelques-uns de ceux-ci ont néanmoins été déposés chez un notaire par la suite ${ }^{21}$. On peut donc considérer qu'une proportion importante des sociétés de traite se retrouve dans les minutiers des notaires et que la documentation utilisée permet de tracer un portrait valide de cet aspect du commerce des fourrures.

De ce portrait, sont exclues certaines formes d'exploitation conjointe du commerce. Dans leur structure, plusieurs associations s'apparentaient aux sociétés anonymes. Elles résultaient d'obligations solidaires; sans spécifier explicitement les fonctions de chacun, ni la durée de l'entente ni la répartition des profits, ces contrats rendaient les débiteurs conjointement responsables de la dette contractée. D'autres sociétés n'ont été que des ententes verbales, comme tend à le montrer le préambule du contrat de société de Guillaume Dupont et de Jacques Rouillard: les deux hommes s'étaient associés verbalement, mais comme Dupont était tombé malade, ils décidèrent «Pour la Seurete de Leurd. Societte (de) Passer un acte qui Explique Leur Jntention afin d'Eviter Les Difficultés qui pourroit arriver faute Dud. acte...» ${ }^{22}$

Au total, 202 contrats de société ont été retrouvés dans les répertoires des notaires pour les 46 années étudiées, soit en moyenne 4,4 contrats par année ${ }^{23}$. Ces contrats ont été rédigés ou reçus par des notaires de Montréal: Jean-Baptiste Adhémar ( 75 contrats entre 1715 et 1753, $37,1 \%$ de l'ensemble), Michel LePallieur (48 entre 1715 et 1731, 23,8\%), Louis-Claude Danré de Blanzy (20 entre 1738 et 1748, 9,9\%), François LePallieur (13 entre 1733 et 1737, 6,4\%), François Simonnet (13 entre 1740 et $1759,6,4 \%$ ), Jacques David (10 entre 1720 et 1726 , $4,9 \%$ ), Pierre Raimbault (9 entre 1718 et 1726, 4,4\%) et quelques autres qui ne reçurent qu'un tout petit nombre de contrats ${ }^{24}$. On remarque une grande concentration des contrats, deux notaires ayant rédigé les $3 / 5$ des contrats $(60,9 \%)$. Bien plus, dans la grande majorité des cas, un seul notaire reçoit la plupart des contrats d'une année donnée et pour

21 Le contrat de société liant Nicolas-Joseph de Noyelles, commandant au fort Saint-Philippe des Miamis, à François et Pierre Roy est passé au fort même le 24 mai 1728 et déposé par François Roi chez Jean-Baptiste Adhémar le 5 septembre suivant. Une société regroupant de Croisille de Courtemanche, Jacques Rouillard et Louis Prudhomme pour le poste de LaBaye fut passée à Michilimakinac à une date non indiquée et déposée chez François LePallieur le 13 septembre 1735. De même, les Associés de la Mer de l'Ouest se sont formés à Michilimakinac le 9 août 1733 et leur contrat fut déposé chez C.-C.-J. Porlier le 22 mai suivant. Archives nationales du Québec à Montréal (ANQ-M), CN0601-0003, J.-B. Adhémar, 5 sept. 1728, no 2583; CN06010259, F. LePallieur, 13 sept. 1735, no 957; CN0601-0329, C.-C.-J. Porlier, 22 mai 1734, no 97.

22 ANQ-M, CN0601-0003, J.-B. Adhémar, 6 juillet 1729 , no 2864 . ANQ-M, CN0601-0260, M. LePallieur, 8 mars 1718, annulé le 3 août suivant, no 573; CN06010003 , J.-B. Adhémar, 15 mars 1724, annulé le 24 mai suivant, no $1128 ; 12$ avril 1725, annulé le 27 mai suivant, no 1458; ler juillet 1729 , annulé le 7 juillet suivant, no 2859 ; 17 avril 1732 , annulé le 23 mai suivant, no $5539 ; 15$ janvier 1735 , annulé le 5 mai suivant, no 6563 .

24 Ces notaires sont: Gervais Hodiesne (4), Henri Bouron (2 contrats), Charles-René Gaudron de Chèvremont (2), Pierre Panet (2), Claude-Cyprien-Jacques Porlier (2), Nicolas-Auguste Guillet de Chaumont (1), Joseph-Charles Raimbault (1) et André Souste (1). 
plusieurs années consécutives: M. LePallieur d'abord, puis J.-B. Adhémar suivi de F. LePallieur, de L.-C. Danré de Blanzy et de F. Simonnet (voir le tableau 1). Aucun contrat n'a été retrouvé chez les notaires des régions rurales du gouvernement de Montréal $^{25}$.

TABLEAU 1

Répartition des contrats de société pour l'exploitation des postes des Pays-d'en-haut, par année et par notaire (1715-1760)

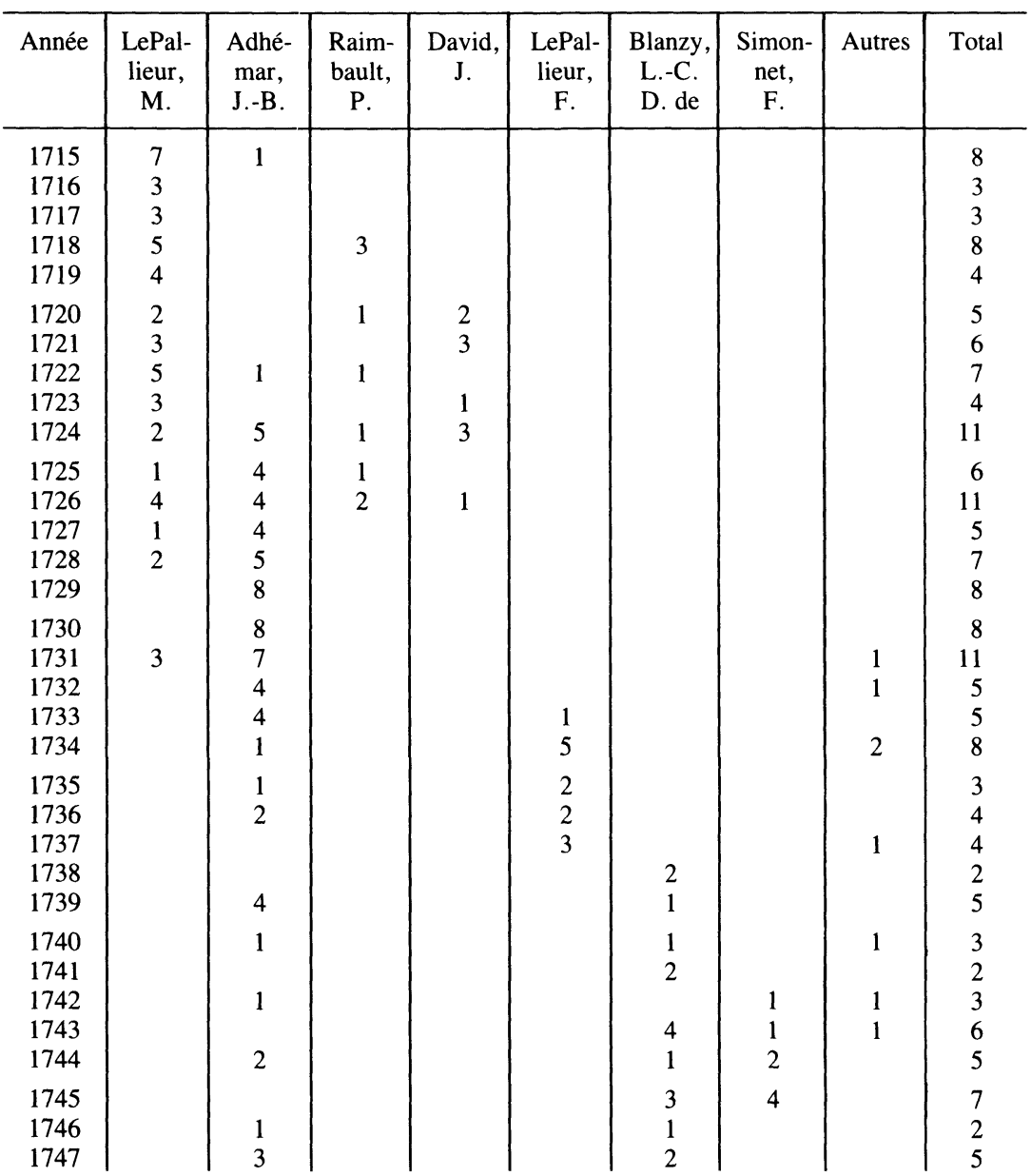

25 La recherche n'a pas encore porté sur les greffes des notaires ayant exercé à TroisRivières ou à Québec; étant donné le lieu de résidence de quelques officiers de poste, il est possible que l'on trouve quelques contrats à ces endroits. 


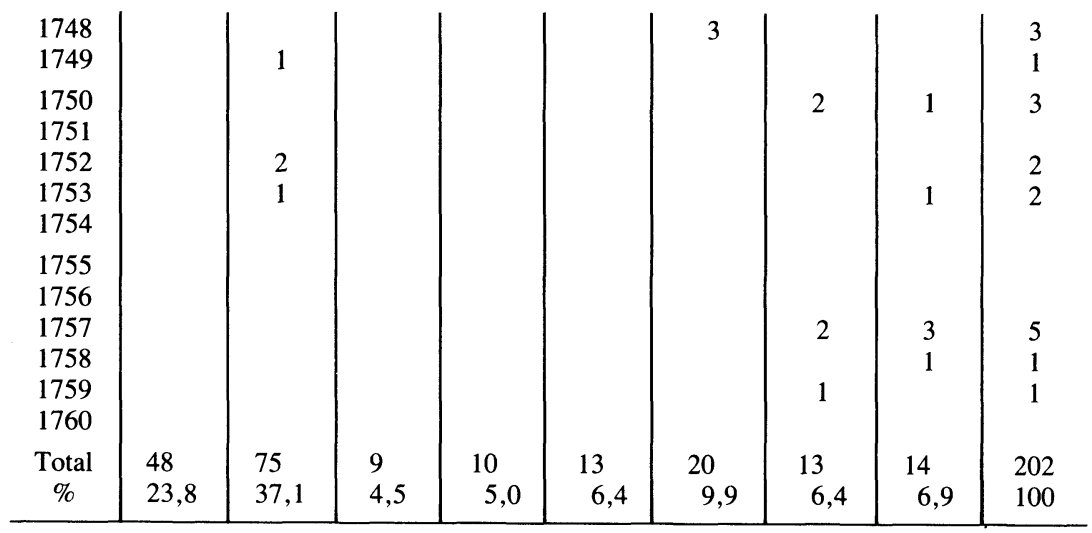

Source: ANQ-M, CNO601, J.-B. Adhémar, H. Bouron, L.-C. Danré de Blanzy, J. David, C.-R. Gaudron de Chèvremont, N.-A. Guillet de Chaumont, G. Hodiesne, F. LePallieur, M. LePallieur, P. Panet, C.-C.-J. Porlier, J.-C. Raimbault, P. Raimbault, F. Simonnet et A. Souste.

Selon Savary des Brûlons, plusieurs éléments doivent faire partie d'un contrat de société: la mention du capital fourni, la durée prévue, le partage des profits ou des pertes, la défense de commercer en dehors de la société de traite, les modalités prévues pour terminer la société ou la continuer en cas de décès de l'un des associés, et quelques autres particularités comme une aumône aux pauvres. Certains de ces éléments, comme la durée, le partage des profits et le moment de ce partage, de même que la défense de commercer en dehors de la société, sont presque toujours présents dans les contrats de société de traite. D'autres, comme l'aumône aux pauvres et les modalités à suivre en cas de décès de l'un des associés, font rarement partie des clauses du contrat. Le but poursuivi par la société et le lieu du commerce sont la plupart du temps clairement indiqués. Il faut dire que les contrats de société pour la traite des fourrures ont évolué au cours des 35 années étudiées. Ils ont acquis, en général, une plus grande précision et un plus grand détail. Les contrats des années 1710 sont, pour la plupart, beaucoup moins élaborés que ceux des années 1730.

Le contenu des contrats a été influencé par plusieurs facteurs. Ils diffèrent d'un notaire à l'autre: les contrats que rédigent Jacques David ou Charles-René Gaudron de Chèvremont comprennent beaucoup moins de détails que ceux de Jean-Baptiste Adhémar ou de François LePallieur. Cette influence ne peut cependant pas être considérée comme déterminante. On peut remarquer une évolution vers une plus grande précision chez Adhémar lui-même. Le but poursuivi par la société constitue un facteur très important. Les associés qui s'apprêtent à exploiter exclusivement un poste pendant 3 à 5 ans apportent un plus grand soin à la rédaction du contrat que ceux qui s'associent pour faire valoir un congé 
d'un canot, le temps que les marchandises soient écoulées. L'importance du poste à exploiter et le nombre des associés entrent aussi en ligne de compte.

Deux contrats de 1737 illustrent ces arguments. Le 31 juillet, deux voyageurs de Montréal, Étienne Petit de Boismorel et Pierre Lefebvre Duchouquet s'associent pour le commerce des Illinois. Leur contrat, passé devant Gaudron de Chèvremont, donne la provenance de l'investissement et prévoit le remboursement des fournisseurs avant le partage, sans plus ${ }^{26}$. Le 27 juin précédent, François LePallieur avait rédigé le contrat de société de trois marchands voyageurs, Nicolas Roze, JeanBaptiste Garrau et Thomas Blondeau, qui s'associaient pour exploiter le poste de LaBaye à ferme pendant 3 ans. Le contrat est minutieux: il comprend les clauses habituelles, prévoit la vérification des factures et l'arbitrage en cas de contestation, décrit les relations avec le marchandéquipeur, incluant une procuration, prescrit les paiements à faire pour la ferme du poste et indique les modalités à suivre à la dissolution de la sociétét ${ }^{27}$.

\section{ÉVOLUTION DES CONTRATS DE SOCIÉTÉ}

On peut distinguer trois périodes dans l'évolution des contrats de société, selon la fréquence des contrats, la durée prévue des sociétés, la précision des destinations mentionnées et la participation des commandants de poste. La première, de remise en place et de rodage du système, dure de 1715 à 1721; la seconde, caractérisée par la participation directe des commandants, s'étend de 1722 à 1734; la troisième, de 1735 à 1760 , est marquée par la perte d'importance du contrat de société et, peut-être, de la société de traite. Le tableau 2 témoigne de cette évolution.

\section{TABLEAU 2}

Répartition des contrats de société pour l'exploitation des postes des pays-d'en-haut, par année et par nombre d'associés (1715-1760)

\begin{tabular}{|c|c|c|c|c|c|}
\hline \multirow[t]{2}{*}{ Année } & \multicolumn{4}{|c|}{ Nombre d'associés } & \multirow[t]{2}{*}{ Total } \\
\hline & 2 & 3 & 4 & $5-7$ & \\
\hline 1715 & $5(2 \mathrm{~m})$ & $3(1 \mathrm{~m})$ & & & $8(3 m)$ \\
\hline 1716 & & $1(1 \mathrm{~m})$ & & & $3(1 \mathrm{~m})$ \\
\hline 1717 & 3 & & & & 3 \\
\hline 1718 & & 2 & & & 8 \\
\hline 1719 & $4(1 \mathrm{~m})$ & & & & $4(1 \mathrm{~m})$ \\
\hline 1720 & 4 & & & 1 & 5 \\
\hline 1721 & 3 & 3 & & & 6 \\
\hline $1715-1721$ & $27(3 m)$ & $9(2 \mathrm{~m})$ & & 1 & $37(5 \mathrm{~m})$ \\
\hline
\end{tabular}

\footnotetext{
26 ANQ-M, CN0601-0089, C.-R. Gaudron de Chèvremont, 31 juillet 1737, no 277.

27 ANQ-M, CN0601-0259, F. LePallieur, 27 juin 1737, no 1858.
} 


\begin{tabular}{|c|c|c|c|c|c|}
\hline 1722 & 6 & 1 & & & 7 \\
\hline 1723 & 4 & & & & 4 \\
\hline 1724 & 8 & 1 & 2 & & 11 \\
\hline 1725 & 5 & 1 & & & 6 \\
\hline 1726 & 5 & 5 & 1 & & 11 \\
\hline 1727 & $4(1 \mathrm{~m})$ & & 1 & & $5(1 \mathrm{~m})$ \\
\hline 1728 & $4(1 \mathrm{r})$ & 2 & 1 & & $7(1 \mathrm{r})$ \\
\hline 1729 & $6(1 \mathrm{~m})$ & 2 & & & $8(1 \mathrm{~m})$ \\
\hline 1730 & 7 & 1 & & & 8 \\
\hline 1731 & 8 & $3(1 \mathrm{~m})$ & & & $11(1 \mathrm{~m})$ \\
\hline 1732 & $4(1 \mathrm{~m})$ & & & & $5(1 \mathrm{~m})$ \\
\hline 1733 & 2 & $3(1 \mathrm{~m})$ & & & $5(1 \mathrm{~m})$ \\
\hline 1734 & 5 & 2 & & 1 & 8 \\
\hline $1722-1731$ & $68(4 \mathrm{mr})$ & $22(2 \mathrm{~m})$ & 5 & 1 & $96(6 \mathrm{mr})$ \\
\hline 1735 & 1 & 1 & & 1 & 3 \\
\hline 1736 & 3 & 1 & & & 4 \\
\hline 1737 & 2 & 2 & & & 4 \\
\hline 1738 & 1 & & $1(1 r)$ & & $2(1 r)$ \\
\hline 1739 & 4 & 1 & & & 5 \\
\hline 1740 & 1 & 2 & & & 3 \\
\hline 1741 & 1 & 1 & & & 2 \\
\hline 1742 & 3 & & & & 3 \\
\hline 1743 & 4 & 1 & & 1 & 6 \\
\hline 1744 & 3 & 1 & 1 & & 5 \\
\hline 1745 & 5 & 2 & & & 7 \\
\hline 1746 & 1 & & 1 & & 2 \\
\hline 1747 & $4(1 \mathrm{~m})$ & & 1 & & $5(1 \mathrm{~m})$ \\
\hline 1748 & 1 & 1 & 1 & & 3 \\
\hline 1749 & 1 & & & & 1 \\
\hline 1750 & 3 & & & & 3 \\
\hline 1751 & & & & & 0 \\
\hline 1752 & 2 & & & & 2 \\
\hline 1753 & 2 & & & & 2 \\
\hline 1754 & & & & & 0 \\
\hline 1755 & & & & & 0 \\
\hline 1756 & & & & & 0 \\
\hline 1757 & 3 & 2 & & & 5 \\
\hline 1758 & 1 & & & & 1 \\
\hline 1759 & 1 & & & & 1 \\
\hline 1760 & & & & & 0 \\
\hline $1735-1760$ & $47(1 \mathrm{~m})$ & 15 & $5(1 r)$ & 2 & $69(2 \mathrm{mr})$ \\
\hline Total & $142(8 \mathrm{mr})$ & $46(4 \mathrm{~m})$ & $10(1 \mathrm{r})$ & 4 & $202(13 \mathrm{mr})$ \\
\hline
\end{tabular}

Notes: Les lettres et les chiffres entre parenthèse indiquent les contrats manquants (m) ou à la restauration (r) et leur nombre.

Dans les contrats de 5 à 7 associés sont compris un contrat de 5 associés en 1720 , un de 6 en 1734 et un de 7 associés en 1735 et 1743 .

Les contrats de 1757 comprennent un contrat de société générale pour les «affaires» des Paysd'en-haut entre le munitionnaire Joseph Cadet et le négociant Jacques Porlier (CNO601-0308, P. Panet, 2 avril 1757, no 373.)

Source: ANQ-M, CNO601, J.-B. Adhémar, H. Bouron, L.-C. Danré de Blanzy, J. David, C.R. Gaudron de Chèvremont, N.-A. Guillet de Chaumont, G. Hodiesne, F. LePallieur, M. LePallieur, P. Panet, C.-C.-J. Porlier, J.-C. Raimbault, P. Raimbault, F. Simonnet et A. Souste. 
Les sept années qui suivent le rétablissement des congés en 1715 constituent une période de remise en place des divers éléments du commerce des fourrures. Pour cette période, on a relevé dans les répertoires 37 contrats de société, soit une moyenne annuelle de 5,3 contrats. Ces contrats représentent $18,3 \%$ de l'ensemble. Cinq d'entre eux n'ont pas été retrouvés. Les 32 autres sont caractérisés par le manque de précision des destinations qui y sont inscrites. Dans la moitié des cas, le notaire a écrit «les Outaouais» ou «le pays des Outaouais», ou «les pays d'en haut». Par contre, la mise de fonds en marchandises de chacun des associés est fréquemment inscrite à l'acte, sans doute afin d'assurer le remboursement de ces sommes à la fin de la société. Ce renseignement n'est pas utilisé pour déterminer la répartition des profits ou des pertes entre les associés, qui est plutôt décidée selon d'autres critères.

Les trois quarts de ces sociétés sont composées de 2 associés; 7 autres sont formées de 3 personnes et une dernière regroupe 5 partenaires. Les associés se partagent également les profits ou les pertes, sauf dans 3 cas, où la moitié des profits revient à l'un des partenaires et l'autre moitié aux deux autres. On retrouve un commandant de poste dans seulement 7 de ces sociétés, un officier des troupes de la marine dans 7 autres. La participation directe des officiers n'est donc pas encore un élément prédominant de la société de traite. En réalité, les commandants ne sont pas toujours intéressés à participer directement au commerce des fourrures. Jean-Baptiste Bissot de Vinsenne, par exemple, commandant au pays des Miamis et des Ouiatanons, s'était, le 8 mars 1718, associé à Jacques Hubert-Lacroix pour exploiter deux permissions accordées à de Vinsenne. Un mois et demi plus tard, HubertLacroix fit annuler la société puisque de Vinsenne était parti sans l'attendre et avait vendu les permissions à un autre ${ }^{28}$.

Les congés que détiennent les associés influencent fortement la durée des contrats de cette première période: la société ne doit durer qu'un hivernement ou le temps d'écouler les marchandises de traite. Font exception à cette règle quatre sociétés seulement, dont la durée prévue est de deux ans et plus. Dans l'un de ces 4 cas, l'un des associés est officier «au service du roi» ${ }^{29}$; dans les 3 autres, il est commandant de poste ${ }^{30}$.

Pour la deuxième période, de 1722 à 1734 , le nombre annuel de contrats retrouvés dans les répertoires varie entre 4 et 12; la moyenne

\footnotetext{
28 ANQ-M, CN0601-0260, M. LePallieur, 8 mars 1718, no 3045.

29 Martin Rémy de Montmidy, officier pour le poste de Rivière-Saint-Joseph en 1717. Ibid., 26 août 1717 , no 269 l.

30 Jean-Baptiste de Saint-Ours Deschaillons, en 1717, est prêt à aller commander au poste qu'il «plaira (au gouverneur) Luy départir et destiner» au pays des Outaouais; en 1721, il est détaché pour aller commander au poste de Kaministiquia. Louis Liénard de Beaujeu, en 1719, est commandant au poste de Michilimakinac. Ibid., 20 juin 1717, no 2556; 29 mai 1719, no 3224; 24 mars 1721 , no 3573 .
} 
annuelle de 7,4 représente bien l'ensemble. Un total de 96 contrats ont été passés devant notaire ou reçus par eux au cours de ces 13 années. En excluant les 6 contrats manquants ou à la restauration, il reste 90 contrats qui témoignent des caractéristiques des sociétés de cette période.

L'évolution des sociétés a été fortement influencée par les décisions des autorités métropolitaines et coloniales. Le nombre des sociétés de même que les clauses qu'elles contiennent sont souvent la conséquence de ces décisions. Annoncée en 1720, appliquée en 1723, l'abolition des congés a duré jusqu'en 1728. Elle a permis la mise sur pied d'un système de permissions, nécessaires pour l'approvisionnement des postes des Pays-d'en-haut. Jusqu'en 1734, les commandants des postes et les officiers ont profité de ces permissions pour participer directement au commerce des fourrures: un peu plus du tiers des sociétés formées durant ces années (33 ou 36,3\%) comptent le commandant du poste comme associé. Les modalités ne sont pas neuves, ayant été établies au cours de la période précédente, mais elles sont plus largement répandues. Durant ces années, le contrat atteint le maximum de détail et de précision.

Les destinations inscrites au contrat sont précises dans la plupart des cas; seuls 18 contrats $(20,0 \%)$ donnent les mentions générales «les pays d'en haut» (17 contrats) ou «les Outaouais» ( 1 contrat) comme destination. A l'inverse de la période précédente, la mise de fonds des associés est de moins en moins fréquemment indiquée; les contrats prévoient plutôt que les marchandises seront prises chez un marchand et qu'elles feront l'objet d'une obligation solidaire. Ces contrats sont pour une plus longue durée. Un peu moins de la moitié $(44,4 \%)$ sont pour un hivernement ou moins, le tiers $(32,2 \%)$ sont pour 2 ans et plus, l'un de ces derniers étant même pour 9 ans, 4 dureront aussi longtemps que l'associé aura le poste en question et les autres sont de durée inconnue.

Comme pour la période précédente, les sociétés passées devant notaire au cours de ces treize années sont en majorité composées de 2 associés $(76,7 \%)$. Dans ces sociétés, les profits sont répartis à parts égales, à quelques exceptions près où les proportions sont différentes: deux tiers pour l'un des associés, commandant de poste ou officier, et un tiers pour l'autre ( 3 cas) ou trois quarts pour l'un et un quart pour l'autre ( 2 cas). Les autres sociétés regroupent 3 personnes ( 21 contrats, $23,3 \%$ de cette période) et même, fait nouveau, 4 personnes (5 contrats) et plus ( 1 contrat). Dans ces sociétés, la répartition des profits ou des pertes est intéressante: elle est à part égale (un tiers ou un quart chacun, selon le cas) dans 12 cas; pour les autres, elle est inégale, la plus fréquente étant la moitié pour l'un des associés et l'autre moitié pour les deux ou trois autres. Dans la plupart des cas de distribution inégale, 
l'un des partenaires est commandant de poste ${ }^{31}$; il l'est dans la moitié des cas de répartition égale.

D'autres précisions font partie de ces contrats, par lesquels les associés tentent de prévoir les difficultés éventuelles. Les contrats se terminent de plus en plus fréquemment par une clause d'arbitrage: deux marchands seraient nommés par les associés pour régler un éventuel différend lors de la dissolution de la société. On indique de plus en plus souvent que les associés seront nourris aux frais de la société, tant au cours du voyage que durant le séjour au poste de destination. On stipule également dans certains contrats les quantités d'effets que la société sera tenue de monter ou de fournir sans frais au commandant du poste. On définit aussi, dans quelques contrats, la propriété des présents offerts par les Amérindiens: lorsque le commandant se les réserve, il doit payer à la société, selon des termes fixés d'avance, les produits qu'il y prend pour rendre la politesse aux Amérindiens. On prévoit aussi que les associés pourront percevoir leurs créances antérieures, sans toutefois se servir de ce revenu pour faire la traite pour eux-mêmes. Dans deux cas, Nicolas-Antoine Coulon de Villiers en 1725 à Rivière-Saint-Joseph ${ }^{32}$ et Louis Denys de la Ronde en 1734 à Chagouamigon ${ }^{33}$, des modalités précises sont inclues au contrat, en faveur du fils du commandant.

Pour la troisième période, de 1735 à 1760,69 contrats inventoriés, soit $34,2 \%$ de l'ensemble. Le nombre annuel de contrats s'élève rarement à plus de 5; la moyenne annuelle, de 2,6 contrats, est la moins élevée des trois périodes. Après 1750, il n'y a pas de contrats une année sur deux. Comme au cours des périodes précédentes, la grande majorité des 67 sociétés dont le contrat a été retrouvé $(68,7 \%)$ comptent deux associés. Quant aux autres sociétés, 15 réunissent 3 personnes et les 6 autres en regroupent 4 et plus. A quelques exceptions près, les profits sont répartis également entre les associés. Le nombre peu élevé de contrats durant ces années ne provient donc pas d'une concentration d'associés dans un plus petit nombre de sociétés. Il s'explique en partie par la durée plus longue de ces contrats: 22 contrats portent sur 3 ans, 2 sur 2 ans $^{34}, 1$ sur 5 ans $^{35}, 1$ sur 6 ans $^{36}$ et un autre sur 9 ans $^{37}$. En enlevant les 10 cas dont la durée est inconnue, les 3 autres dont elle est

31 Les contrats passés par Jean Lemire Marsolet, pour sa société avec Constant Le Marchand de Lignery, prévoient la moitié des profits pour cette société et l'autre moitié pour l'autre associé. Comme la société de Lignery-Lemire Marsolet est elle-même à demi-profit, chacun des deux hommes obtient le quart de la nouvelle société. ANQ-M, CN0601-0003, J.-B. Adhémar, 8 avril 1826, no 1724; ibid., 26 avril 1726, no 1770; ibid., 23 mai 1726, no 1854.

32 Ibid., 27 mai 1725 , no 1525.

33 ANQ-M, CN0601-(0259, F. LePallieur, 14 mai 1734, no 221.

34 ANQ-M, CN0601-(0108, L.-C. Danré de Blanzy, 6 mai 1739, no 160; CN0601-0003, J.-B. Adhémar, 17 juin 1752, no 1449.

35 ANQ-M, CN0601-(0003, J.-B. Adhémar, 19 juin 1739, no 7682.

36 ANQ-M, CN0601-(0308, P. Panet, 8 mai 1758, no 822.

37 ANQ-M, CN0601-(0108, L.-C. Danré de Blanzy, 23 juillet 1749, no 2957. 
en fonction du temps d'assignation au poste en question et un dernier où l'association est faite pour la montée seulement ${ }^{38}$, il en reste 26 qui portent sur un hivernement ou une saison.

En général, les clauses des contrats sont les mêmes qu'auparavant. Certaines modifications indiquent toutefois un changement de situation. Les mises de fonds de la part des associés se trouvent indiquées plus fréquemment, le nom des équipeurs paraît plus souvent dans les contrats, ainsi que les conditions de l'approvisionnement en marchandises. La clause d'arbitrage que l'on y retrouve généralement prévoit à quelques reprises une forte pénalité, jusqu'à 1000 livres, pour celui qui ne la respecterait pas. Le changement le plus significatif touche une des catégories d'associés. Pour des raisons difficiles à cerner, les commandants de poste, sauf exception, ne participent plus directement au commerce des fourrures. On ne retrouve que 7 contrats de société où l'un des partenaires est le commandant, 3 où il est un officier. Le contraste est frappant avec la période précédente. On assiste donc à une forte diminution d'importance du contrat de société après 1735 .

\section{STATUT PROFESSIONNEL DES ASSOCIÉS}

En plus de retracer l'évolution de cette partie du commerce des fourrures, les contrats de société permettent de mieux connaitre le statut professionnel de ceux qui y participaient et l'importance relative des groupes professionnels auxquels ils appartenaient. Pour les fins de cette analyse, on a utilisé les 455 mentions d'associé relevées dans les contrats, sans s'attarder à déterminer par jumelage des contrats le nombre d'individus impliqués. Les personnes qui ont fait partie de plusieurs sociétés sont donc l'objet d'autant de mentions. Cette méthode de calcul et d'analyse a été préférée à celle du jumelage parce qu'elle accorde aux renseignements recueillis une pondération appropriée. Ces calculs ne tiennent cependant pas compte de la durée des contrats.

On constate que l'éventail des professions retrouvées dans les contrats n'est pas très étendu: au total, 23 professions différentes sont représentées (voir le tableau 3). Le regroupement de certaines d'entre elles se fait facilement. Habitants-voyageurs, interprètes, interprètevoyageur et sergent-voyageur sont groupés aux voyageurs, tout comme les négociants et les marchands-négociants le sont au marchand. Ces groupes professionnels ont ensuite été regroupés en quatre catégories pour tenir compte de l'intérêt de chacun et de son rang social. La première comprend les commandants des postes et les officiers militaires, auxquels on a ajouté les écuyers, ces nobles sans distinction particulière. L'intérêt des personnes de cette catégorie pour le commerce des fourrures découle de leur fonction de commandement. Dans la deuxième

38 ANQ-M, CN0601-0372, F. Simonnet, 5 avril 1757, no 196. 
catégorie, on retrouve les marchands, les voyageurs et les marchandsvoyageurs; le commerce des fourrures constitue le fondement même de leur profession. La troisième catégorie est un fourre-tout où l'on trouve des habitants, des seigneurs et des hommes de métier. La quatrième catégorie est celle des "profession non indiquée».

La lecture du tableau 3 impose une première constatation: le pourcentage des mentions d'associés qui ne sont pas suivies de la profession est très élevé: $35,8 \%$. Comme il est fort peu probable qu'officiers et écuyers n'aient pas indiqué leur occupation ou leur titre, ces contractants sans profession donnée ont été assimilés au groupe des marchands, voyageurs ou marchands-voyageurs. Ce qui amène une deuxième constatation: même en lui ajoutant les écuyers, dont le rôle dans la traite s'apparente plutôt à celui du voyageur et du marchand-voyageur, le groupe des commandants de poste et officiers militaires forme moins du quart du nombre total des associés. Si l'on exclut les écuyers, ce groupe ne fait que $14,9 \%$ de l'ensemble et se retrouve encore plus concentré dans la deuxième période identifiée (1722-1734).

Le groupe des commandants de poste et des officiers militaires comprend des hommes dont la participation directe au commerce des fourrures est de durée variable. Pierre Robinau de Portneuf, commandant-en-second aux Sioux en 1731, ne contracte qu'une sociétét ${ }^{39}$; de même que Charles-François Tarieu de Lapérade, officier des troupes de la marine ${ }^{40}$. Jean-Baptiste de Saint-Ours Deschaillons, écuyer et

TABLEAU 3

Répartition des associés par groupe professionnel et par année

(1715-1760)

\begin{tabular}{|c|c|c|c|c|c|c|c|c|c|c|c|}
\hline \multirow[b]{2}{*}{ Année } & \multicolumn{5}{|c|}{ Commandants et officiers } & \multicolumn{5}{|c|}{ Marchands et voyageurs } & \multirow[b]{2}{*}{ TOT } \\
\hline & $\mathrm{COM}$ & OFF & ECU & S-T & MAR & VOY & $M-V$ & S-T & AUT & $\mathrm{N}-\mathrm{I}$ & \\
\hline 1715 & 1 & 2 & 1 & 4 & & & & & 2 & 6 & 12 \\
\hline 1716 & 2 & & & 2 & & 1 & & 1 & 1 & & 4 \\
\hline 1717 & & 2 & & 2 & & 1 & & 1 & & 3 & 6 \\
\hline 1718 & 2 & 1 & 1 & 4 & & 10 & & 10 & & 4 & 18 \\
\hline 1719 & 1 & & & 1 & 1 & 2 & & 3 & & 2 & 6 \\
\hline 1720 & & & 1 & 1 & 4 & 2 & & 6 & & 6 & 13 \\
\hline 1721 & 1 & 2 & 1 & 4 & 1 & 2 & & 3 & & 8 & 15 \\
\hline $15-21$ & 7 & 7 & 4 & 18 & 6 & 18 & & 24 & 3 & 29 & 74 \\
\hline 1722 & 1 & 5 & & 6 & 1 & 3 & 1 & 5 & 1 & 3 & 15 \\
\hline 1723 & 2 & 2 & & 4 & 1 & 1 & & 2 & & 2 & 8 \\
\hline 1724 & 6 & & 1 & 7 & & 5 & & 5 & & 15 & 27 \\
\hline
\end{tabular}

39 ANQ-M, CN0601-0260, M. LePallieur, 28 juin 1731, no 4804.

40 ANQ-M, CN0601-0108, L.-C. Danré de Blanzy, 6 juin 1743, no 1788. 
OFFICIERS ET MARCHANDS: LES SOCIÉTÉS DE...

\begin{tabular}{|c|c|c|c|c|c|c|c|c|c|c|c|}
\hline 1725 & 2 & & & 2 & 2 & 6 & & 8 & & 3 & 13 \\
\hline 1726 & 5 & 1 & 3 & 9 & 3 & 3 & 3 & 9 & 2 & 9 & 29 \\
\hline 1727 & 2 & 1 & 1 & 4 & & & & & & 6 & 10 \\
\hline 1728 & 4 & & & 4 & & 3 & & 3 & & 9 & 16 \\
\hline 1729 & 3 & 1 & & 4 & 1 & 8 & & 9 & 1 & 2 & 16 \\
\hline 1730 & 2 & & 4 & 6 & 1 & & & 1 & & 10 & 17 \\
\hline 1731 & 2 & & 5 & 7 & 1 & 3 & & 4 & & 11 & 22 \\
\hline 1732 & & & 1 & 1 & & & & & & 8 & 9 \\
\hline 1733 & & & 1 & 1 & 2 & & & 2 & & 7 & 10 \\
\hline 1734 & 4 & & 1 & 5 & 3 & & 4 & 7 & 1 & 9 & 22 \\
\hline $22-34$ & 33 & 10 & 17 & 60 & 15 & 32 & 8 & 55 & 5 & 94 & 214 \\
\hline 1735 & 1 & & 2 & 3 & 1 & & 2 & 3 & 1 & 5 & 12 \\
\hline 1736 & & & & & 1 & 3 & 2 & 6 & 1 & 2 & 9 \\
\hline 1737 & & & & & & 2 & 7 & 9 & & 1 & 10 \\
\hline 1738 & & & & & & 2 & & 2 & & & 2 \\
\hline 1739 & 1 & & & 1 & 3 & 3 & 1 & 7 & 1 & 2 & 11 \\
\hline 1740 & 1 & & & 1 & & 1 & 3 & 4 & 2 & 1 & 8 \\
\hline 1741 & & & 3 & 3 & 1 & 1 & & 2 & & & 5 \\
\hline 1742 & & & & & & 2 & 2 & 4 & & 2 & 6 \\
\hline 1743 & & 1 & 2 & 3 & 3 & 4 & 1 & 8 & & 7 & 18 \\
\hline 1744 & & 1 & 2 & 3 & 2 & 3 & 5 & 10 & & & 13 \\
\hline 1745 & 1 & & & 1 & 7 & 5 & & 12 & 2 & 1 & 16 \\
\hline 1746 & & & 1 & 1 & & 3 & & 3 & 1 & 1 & 6 \\
\hline 1747 & & & 2 & 2 & 1 & & & 1 & 1 & 6 & 10 \\
\hline 1748 & 1 & & 1 & 2 & & & & & & 7 & 9 \\
\hline 1749 & & & & & 1 & & & 1 & & 1 & 2 \\
\hline 1750 & & & 2 & 2 & 1 & & & 1 & 1 & 2 & 6 \\
\hline 1751 & & & & & & & & & & & . \\
\hline 1752 & & & & & 1 & & 1 & 2 & & 2 & 4 \\
\hline 1753 & & & & & 3 & & 1 & 4 & & & 4 \\
\hline 1754 & & & & & & & & & & & 0 \\
\hline 1755 & & & & & & & & & & & 0 \\
\hline 1756 & & & & & & & & & & & 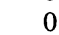 \\
\hline 1757 & 2 & & & 2 & 6 & & 3 & 9 & 1 & & 12 \\
\hline 1758 & & & & & 2 & & & 2 & & & 2 \\
\hline 1759 & & 2 & & 2 & & & & & & & 2 \\
\hline 1760 & & & & & & & & & & & 0 \\
\hline $35-60$ & 7 & 4 & 15 & 26 & 33 & 29 & 28 & 90 & 11 & 40 & 167 \\
\hline Total & 47 & 21 & 36 & 104 & 54 & 79 & 36 & 169 & 19 & 163 & 455 \\
\hline$\%$ & 10,3 & 4,8 & 7,8 & 22,9 & 11,8 & 17,7 & 7,9 & 37,3 & 4,1 & 35,6 & \\
\hline
\end{tabular}

Notes: Les contrats manquants ne sont pas inclus dans cette compilation; ils auraient pour effet d'augmenter encore le nombre d'occupations manquantes.

COM: commandants de poste (incluant 3 commandants-en-second, pour 1727, 1731 et 1739); OFF: officiers militaires; ECU: écuyers; MAR: marchands ou marchands-bourgeois (incluant 17 négociants après 1743 et 2 marchands-négociants); VOY: voyageurs (incluant 2 habitants-voyageurs pour 1718 et 1722, 2 interprètes pour 1725 et 1726; 1 interprète-voyageur pour 1729 et 1 sergent-voyageur pour 1736); M-V: marchands-voyageurs (incluant 2 négociants-voyageurs pour 1744, 1 pour 1752 et 1 pour 1753); AUT: autres (6 habitants, 4 seigneurs, 2 interprètes, 2 forgeronstaillandiers, 1 forgeron, 1 taillandier, 1 armurier et 1 bourgeois); N-I: non indiquée; S-T: soustotal; TOT: total.

Source: ANQ-M, CNO601, J.-B. Adhémar, H. Bouron, L.-C. Danré de Blanzy, J. David, C.-R. Gaudron de Chèvremont, N.-A. Guillet de Chaumont, G. Hodiesne, F. LePallieur, M. LePallieur, P. Panet, C.-C.-J. Porlier, J.-C. Raimbault, P. Raimbault, F. Simonnet et A. Souste. 
capitaine d'une compagnie des troupes de la marine, commandait d'abord aux Outaouais, puis, à partir de 1721, à Kaministiquia. Il fait partie de quatre sociétés entre 1715 et 1725: deux pour le pays des Outaouais et deux pour Kaministiquia ${ }^{41}$. Nicolas-Antoine Coulon de Villiers, écuyer et lieutenant d'une compagnie des troupes du détachement de la marine, était commandant au poste de Rivière-Saint-Joseph en 1725 . Il passe deux contrats de société pour ce poste, l'un en 1725 et l'autre en $1728^{42}$. François Lefebvre Duplessis Fabert, écuyer et lieutenant, qui commandait au poste de LaBaye en 1726, conclut 3 sociétés entre 1726 et $1733^{43}$.

Ce groupe est intéressé directement au commerce des fourrures jusqu'au milieu des années 1730. Par la suite, plusieurs commandants de poste préfèrent s'abstenir de participer directement au commerce des fourrures. Aussi, «en vertu des prérogatives qui (leur) sont accordées en qualité de commandant ${ }^{44}$, baillent-ils leur poste à des marchandsvoyageurs en échange d'une somme d'argent et d'autres considérations. Les tribulations de Pierre Gaultier de La Vérendrye sont assez bien connues: elles eurent pour résultat l'affermage de Michipicoton en 1735 et de tous les postes de l'Ouest en 1738, puis encore en 1747. D'ailleurs, Nicolas-Joseph de Noyelles fit de même lorsqu'il prit le commandement des postes en 1744. Plusieurs autres postes furent aussi l'objet de cette désaffection des commandants pour la participation aux sociétés de commerce: le poste des Ouiatanons à partir de 1733, que commandèrent successivement Charles de Mézières de Lépervanche ${ }^{45}$, Louis Godefroy de Normanville ${ }^{46}$, René Godefroy de Linctôt ${ }^{47}$ et Charles-Joseph de Saint-Ours Deschaillons ${ }^{48}$, celui de la Rivière SaintJoseph où commandèrent Paul-Louis Dazemard de Lusignan ${ }^{49}$ et Nicolas-Antoine Coulon de Villiers ${ }^{50}$, le poste des Miamis lorsqu'il était sous le commandement de Philippe Damours de la Morandière ${ }^{51}$ et celui de LaBaye sous le commandement de Claude-Antoine de Bermen de la Martinière ${ }^{52}$.

41 ANQ-M, CN0601-0260, M. LePallieur, 27 mai 1715, no 2038; 20 juin 1717, no 2556; 24 mars 1721, no 3573; CN0601-0003, 13 juin 1725, no 1546.

42 ANQ-M, CN0601-0003, J.-B. Adhémar, 27 mai 1725, no 1525; 9 mai 1728, no 2413.

43 Ibid., 23 mai 1726, no 1853; 17 avril 1732, no 5539; 29 mai 1733, no 6049.

44 L'expression se retrouve dans quelques contrats, dont la cession du commerce de LaBaye par Claude Bermen de la Martinière. ANQ-M, CN0601-0259, F. LePallieur, 13 juin 1737, no 1814. ferme)

ANQ-M, CN0601-0003, J.-B. Adhémar, 2 juin 1733, no 6066, conventions (bail à

ANQ-M, CN0601-0259, F. LePallieur, 4 juin 1735, no 728, bail à ferme.

47 Ibid., 3 juillet 1736 , no 728 , bail à ferme; 3 juin 1738 , no 2446 , bail à ferme.

48 ANQ-M, CN0601-0089, C.R. Gaudron de Chèvremont, 26 février 1739, no 323, conventions (bail à ferme).

49 ANQ-M, CN0601-0259, F. LePallieur, 18 juin 1735, no 854, bail à ferme.

50 Ibid., 31 mai 1738, no 2414, bail à ferme. ferme).

ANQ-M, CN0601-0089, C.-R. Gaudron de Chèvremont, no 214, conventions (bail à ANQ-M, CN0601-()259, F. LePallieur, 13 juin 1737, no 1814, conventions (bail à ferme). 
L'état actuel de la recherche ne permet pas de déterminer pourquoi ces commandants ont préféré affermer leur poste plutôt que d'entrer en société avec un ou des marchands-voyageurs. On ne peut que supposer que les retours diminuaient et que ces hommes préféraient l'assurance de la ferme aux risques de la participation directe. Les déboires de $\mathrm{La}$ Vérendrye illustreraient bien cette hypothèse.

Le groupe des marchands, voyageurs et marchands-voyageurs est mieux réparti dans le temps que celui des commandants de poste et officiers militaires. Il est aussi plus nombreux, représentant $37,1 \%$ de l'ensemble. Si on lui ajoute tous ceux dont l'occupation n'est pas indiquée au contrat, il s'élève à $72,9 \%$. Et il faudrait y ajouter plusieurs écuyers, comme les frères d'Ailleboust et les frères Dagneau, qui jouent le même rôle qu'eux. Ce groupe d'hommes est le coeur du commerce des fourrures. On ne retrouve certains d'entre eux qu'une seule fois comme associés: François et Pierre Delpech St-Cerny, par exemple, en 1719 et 1726 respectivement ${ }^{53}$. D'autres continuent, année après année, à tirer leur subsistance et à accumuler un certain capital en participant successivement à plusieurs sociétés de commerce.

La carrière de François Augé est intéressante à plusieurs points de vue. Il se dit voyageur en 1724 et en 1726, marchand, voyageur et bourgeois en 1735, marchand, voyageur et négociant en 1743. Entre 1726 et 1745, il fait partie de 4 sociétés différentes (1724, 1726, 1732 et 1743), il achète une permission annuelle de 2 canots (1730) et il obtient le bail à ferme du poste des Ouiatanons (1735 et 1736). Il a commercé aux postes de LaBaye, du Détroit, de Rivière-Saint-Joseph et des Ouiatanons. Louis Hamelin a eu une carrière semblable: se disant marchand ou marchand et voyageur, il a, entre 1721 et 1735 , participé à 6 sociétés différentes $(1721,1726,1728,1731,1734$ et 1735$)$ et fait le commerce aux postes du Détroit, Michipicoton, LaBaye, Nipigon, Kaministiquia et Rivière-Saint-Joseph. On pourrait multiplier les exemples: Louis Gastineau, Paul Guillet, Simon Guillory, Jean-Baptiste Réaume.

Ce sont ces voyageurs ou marchands-voyageurs qui assument la gestion du commerce que font les sociétés de traite. Ils le font «de concert» lorsque plusieurs d'entre eux forment la société. Lorsque l'un des associés est commandant de poste, l'autre, ou les autres, sont chargés de la gestion de la société. Dans quelques contrats, il est clairement indiqué que celui qui conduira les affaires de la société le fera sans interférence de la part des autres associés, même si l'un d'entre eux est officier ou commandant de poste, et qu'il sera absolument maître du commerce $^{54}$. Certains de ces contrats, vers la fin des années 1720 ,

53 ANQ-M, CN0601-0260, M. LePallieur, 11 août 1719, no 3264; 9 août 1726, no 4389.

54 Société entre Jean-Paul Legardeur de Saint-Pierre, René Godefroy de Linctôt et Paul Guillet. Ce dernier est chargé de la gestion. Ibid., 10 sept. 1718, no 3082. 
stipulent que la gestion se fera de concert, mais dans ces cas, il est à peu près sûr que le quotidien du commerce est confié à l'expérience du voyageur ou du marchand-voyageur. Il arrive qu'un contrat dise clairement que le voyageur se conformera, «pour le Service du Roy Et le bien de Leur Societté», aux ordres de son associé-commandant de poste, mais c'est l'exception ${ }^{55}$.

Le rôle des marchands, voyageurs et marchands-voyageurs est donc bien défini: ils ont la charge du commerce. Les commandants de poste et les officiers militaires, lorsqu'ils sont membres d'une société de traite, apportent le privilège de faire le commerce, comme on l'indique parfois dans les contrats, «tien(nent) la main Et donne(nt) main forte à Ce quil ny ait aucun (autre) commerçant Dans Led. poste Directe ${ }^{t}$. ny Jndirect $^{\mathrm{t}} .{ }^{56}$

On retrouve rarement des marchands-équipeurs parmi les associés. On peut établir une liste des principaux marchands-équipeurs, valable pour une bonne partie de la période étudiée, en utilisant les obligations passées devant notaire entre 1725 et 1730 . L'importance de chacun est déterminée par la moyenne annuelle, calculée à partir des sommes inscrites dans les contrats d'obligation. Cette moyenne ne représente qu'une limite inférieure, plusieurs sommes en marchandises n'ayant pas fait l'objet d'obligation devant notaire. Un nombre restreint de ces équipeurs (ou groupes d'équipeurs) ont fourni plus de 15000 livres en moyenne par année: Charles Nolan Lamarque et compagnie (environ 24500 livres en moyenne par année), Pierre Le Ber de Senneville et Toussaint Pothier en compagnie (18 000 livres environ), Ignace Gamelin père et fils (environ 17000 livres) et Pierre de Lestage (15 500 livres environ). D'autres ont fourni entre 7500 livres et 13000 livres: les Charly, Jean-Baptiste et, après sa mort, ses fils (environ 13000 livres en moyenne par année), puis, avec des montants moindres, Anne-Marie Chaspoux, veuve Soumande, et Pierre Chartier (environ 10000 livres en moyenne chacun) et Jean-Baptiste Hervieux (7 500 livres environ).

Les Gamelin, Lestage, Chartier, Chaspoux-Soumande et Hervieux n'ont participé directement à aucune société de traite dont le contrat a été passé devant un notaire ou déposé chez l'un d'entre eux. Pour sa part, Toussaint Pothier a, avant son association avec Senneville, fait partie de trois sociétés, dans lesquelles se trouvait également Antoine Thuné Dufresne: en 1715, les deux hommes s'étaient associés

55 Société entre Jean-Baptiste de Saint-Ours Deschaillons et Pierre Pepin de Laforce. Ibid., 20 juin 1717 , no 2556 .

L'expression est tirée du contrat de société de Constant Le Marchand de Lignery, commandant à Michilimakinac, Jean Lemire Marsolet, Guillaume Cartier et Jean-Baptiste Jutra dit Derosiers, contrat passé devant Jean-Baptiste Adhémar le 8 juin 1728. On en trouve une semblable dans le contrat de Nicolas-Joseph de Noyelles et de Joseph Houtelas, du 7 juillet suivant. ANQM, CN0601-0003, J.-B. Adhémar, 8 juin 1728, no 2443; 7 juillet 1728, no 2536. 
à Pierre Mallet pour le commerce des Outaouais ${ }^{57}$, en 1718, ils faisaient ensemble le commerce au même «endroit» ${ }^{58}$ et, en 1721 , ils se joignaient au commandant du poste de Kaministiquia, Jean-Baptiste de Saint-Ours Deschaillons, pour faire le commerce du poste pendant trois $a^{a n s}{ }^{59}$. Les fils Charly, Jacques et Louis, formèrent chacun une société en 1745 pour le poste des Miamis ${ }^{60}$ et celui de Chagouamigon ${ }^{61}$. Quant à Charles Nolan Lamarque, on ne le retrouve que dans deux sociétés: la première, en 1726, pour l'exploitation du poste de Détroit ${ }^{62}$, et la seconde, en 1743, pour Michilimakinac ${ }^{63}$.

Le rôle de ces marchands se situe à d'autres niveaux. Ils sont d'abord et avant tout les fournisseurs de marchandises de traite, comme l'attestent de multiples obligations et leur nom inscrit à ce titre dans certains contrats de société. De plus, après 1730 , plusieurs contrats décrivent les conditions régissant ces conventions de fourniture de marchandises. Louis Charly Saint-Ange, par exemple, s'engage en 1734 à fournir aux associés Louis Denys de la Ronde et Simon Guillory toutes les marchandises pour l'exploitation du poste de Chagouamigon ${ }^{64}$. Charles Nolan Lamarque et Ignace Gamelin sont les fournisseurs de La Vérendrye durant les années de la découverte de la Mer de l'Ouest, mais ni l'un ni l'autre ne font partie des nombreuses sociétés formées successivement pour l'exploitation de ces postes. Leur rôle se limite à fournir les marchandises nécessaires, à embaucher les hommes lorsqu'aucun des associés n'est à Montréal pour le faire, à voir aux affaires de la société dans la vallée du Saint-Laurent, à faire les avances et les paiements requis pour les gages des engagés et la ferme du poste. Il arrive que, comme procureur de l'un ou de plusieurs des associés, ils voient à leurs affaires particulières. En général, ce rôle revient plutôt à l'épouse de l'associé, qui verra ainsi à la recette des fourrures et aura l'oeil à l'ensemble des opérations.

\section{CONCLUSION}

La société de traite telle qu'elle se trouve dans les contrats notariés n'appartient à aucun des genres décrits par Savary des Brûlons dans son Dictionnaire universel de commerce. Il ne s'agit pas d'une société

\footnotetext{
ANQ-M, CN0601-0260, M. LePallieur, 30 avril 1715, no 2018.

Ibid., 11 juillet 1718 , no 3028 .

Ibid., 24 mars 1721 , no 3573.

60 Société avec Charles-André Barthe pour les ouvrages d'armurerie. ANQ-M, CN06010372, F. Simonnet, 9 juin 1745 , no 218.

61 Société avec Goier Belisle pour l'exploitation d'une forge. Ibid., 28 mai 1745, no 174.

62 Contractée pour une période de 5 ans, cette société regroupe, outre Nolan Lamarque,
} Louis Nolan, Louis Gastineau et Joseph Gouin. ANQ-M, CN0601-0111, J. David, 27 mai 1726, no 1092 .

63 Charles Nolan Lamarque s'associait à Louis Lefevre Auger pour 3 ans, du 1er avril 1744 au même jour de 1747. ANQ-M, CN0601-0372, F. Simonnet, 22 juin 1743, no 202.

64 ANQ-M, CN0601-0259, F. LePallieur, 2 juin 1734, no 366 
en commandite: si, dans plusieurs d'entre elles, l'un des associés fournit seulement son expertise, l'autre ne fournit pas le capital, qui est le plus souvent emprunté à des tiers sous forme de marchandises. De plus, c'est celui qui fournit l'expertise qui est, le plus souvent, «le maitre des affaires» ${ }^{65}$. Il ne s'agit pas vraiment d'une société générale, puisque son objet est spécifique et sa durée limitée. Il s'agit plutôt d'un type de société adapté à la situation, tirant des caractéristiques de chacune des deux autres.

L'analyse des sociétés de traite trouvées dans les archives notariales éclaire d'un jour différent le rôle de chacun des partenaires du commerce des fourrures. Il en ressort nettement que le groupe le plus important pour cette activité est celui des voyageurs et des marchandsvoyageurs. Ils se chargeaient de la gestion du commerce, ils fournissaient un certain investissement dont le total collectif varie dans le temps et, surtout, ils paraissent assurer la continuité des opérations.

Le rôle des commandants de poste et des officiers dans ces sociétés de traite a été exagéré. Ils constituent, bien sûr, un facteur important du commerce et de son évolution. C'est en leur faveur que le système de congés a été modifié de façon officieuse au cours des années 1720; ce sont eux qui, à toutes fins pratiques, détenaient la plupart des postes de traite. Cependant, si l'on se fie aux clauses des contrats de société, ils ne touchaient pas à la traite lorsqu'ils faisaient partie de sociétés, et ils devaient même s'assurer, par clause contractuelle, que les présents des Amérindiens ne seraient pas versés à la société. Bien plus, entre 1734 (ou 1737, si l'on tient compte de la durée stipulée aux contrats) et 1760 , ils cédèrent leur privilège à d'autres contre un paiement fixe. A partir de ce moment, ils n'ont plus sur la traite qu'un rôle de surveillance.

Quant aux marchands-équipeurs, ils font très peu souvent partie de sociétés de traite. Leur rôle, important certes, se borne à fournir les équipements nécessaires et, au plus, à se charger des affaires des sociétés à Montréal. Ce qui se faisait souvent sous l'oeil d'un autre procureur de l'un des associés, généralement son épouse.

A ce stade-ci cle l'enquête, il est difficile de déterminer l'importance des conclusions tirées de cette analyse. Elles clarifient le rôle de chacun des groupes d'associés. Cependant, les sociétés étudiées ne sont qu'une facette du commerce des fourrures, une facette dont on ne peut établir l'importance à moins de reconstituer l'ensemble. Il apparaît de plus en plus clairement que les postes devront être les points d'ancrage de cette reconstitution. De là, on pourra ensuite revenir à Montréal.

\footnotetext{
65 «Cette forme de Société semble être appelée en Commandite, parce que celui qui donne son argent à un autre, qui n'apporte très souvent dans la Société que son industrie, est toujours le maître des affaires, \& en état, pour ainsi dire, de commander \& de faire la loi à son associé». Savary des Brûlons, Dictionnaire, 3, col. 144-145.
} 